\title{
Splenic eumelanin differs from hair eumelanin in C57BL/6 mice
}

\author{
Przemyslaw M. Plonka ${ }^{1 凶}$, Dominika Michalczyk ${ }^{1}$, Malgorzata Popik ${ }^{1}$, \\ Bori Handjiski ${ }^{1,2}$, Andrzej Slominski ${ }^{3}$ and Ralf Paus ${ }^{4}$ \\ ${ }^{1}$ Department of Biophysics, Faculty of Biotechnology, Jagiellonian University, Kraków, Poland; ${ }^{2}$ Center of Bio- \\ medical Research, Department of Internal Medicine, Charité, Humboldt University Berlin, Berlin, Germany; \\ ${ }^{3}$ Department of Pathology and Laboratory Medicine, University of Tennessee, Health Science Center, Memphis, \\ USA; ${ }^{4}$ Department of Dermatology, University Hospital Hamburg-Eppendorf, Hamburg, Germany; \\ ఐe-mail: mieszko@awe.mol.uj.edu.pl
}

Received: 05 April, 2005; revised: 10 May, 2005; accepted: 31 May, 2005 available on-line: 25 June, 2005

\begin{abstract}
The presence of melanin in spleens of black C57BL/6 mice has been known for long. Although its origin and biological functions are still obscure, the relation of splenic melanin to the hair follicle and skin pigmentation was suggested. Here, we demonstrated using for the first time electron paramagnetic resonance spectroscopy that black-spotted C57BL/6 spleens contain eumelanin. Its presence here is a "yes or no" phenomenon, as even in the groups which revealed the highest percentage of spots single organs completely devoid of the pigment were found. Percentage of the spotted spleens decreased, however, with the progress of telogen after spontaneouslyinduced hair growth. The paramagnetic properties of the spleen eumelanin differed from the hair shaft or anagen VI skin melanin. The splenic melanin revealed narrower signal, and its microwave power saturability betrayed more heterogenous population of paramagnetic centres than in the skin or hair shaft pigment. Interestingly, the pigment of dry hair shafts and of the wet tissue of depilated anagen VI skin revealed almost identical properties. The properties of splenic melanin better resembled the synthetic dopa melanin (water suspension, and to a lesser degree - powder sample) than the skin/hair melanin. All these findings may indicate a limited degradation of splenic melanin as compared to the skin/hair pigment. The splenic eumelanin may at least in part originate from the skin melanin phagocyted in catagen by the Langerhans cells or macrophages and transported to the organ.
\end{abstract}

Keywords: EPR, hair cycle, hair follicle, pigmentation, melanin degradation, spin-lattice relaxation

Melanin pigmentation is defined not only by melanogenesis but also by processing and transport of melanin granules from melanocytes to other cell types including but not limited to epidermal and hair follicle keratinocytes (Slominski et al., 2004; 2005). Fourty years ago spleen of C57BL/6 mice was suggested to contain melanin (Weissman, 1967). This hypothesis was substantiated by histological demonstration of a black pigment in selected areas of murine spleen that was negative on histochemical iron (Pearls' Prussian Blue) and lipofuscin (ZiehlNeelsen) tests but positive on bleeching with potassium permanganate + oxalic acid (Weissman, 1967; van der Heijden et al., 1995). Ultrastructural studies also demonstrated that the majority of pigmented spleen cells contained melanosomes with fibrillar matrix, and therefore were defined as melanocytes (van der Heijden et al., 1995). Since normal spleen is a rather unusual site for the presence of melanin in the mammalian organism, it is mandatory to define the nature of the cells responsible for the collection, transport, accumulation and/or synthesis of this pigment in spleen. It is also important to define any correlations between splenic melanization and other physiological processes.

The presence of melanin-producing cells outside the skin has been demonstrated primarly in various organs of lower vertebrates (fish, amphibia and reptilia) where this pigment could be found in melanomacrophages - cells of a histiocytic character (Cicero et al., 1982; 1989; Sichel, 1988; Zuasti et al., 1989; Tsujii \& Seno, 1990; Nakamura et al., 1993). These cells are responsible for the removal of pathogens and by-products of oxygen metabo-

-Presented at the XXXII Winter School, 3-7 March 2005, Zakopane, Poland.

Abbreviations: DPPH, 1,1-diphenyl-2-picrylhydrazyl; EPR, electron paramagnetic resonance; PBS, phosphate-buffered saline. 
lism, as well as for the re-utilization of iron in the hemopoietic system (Sichel, 1988; Zuasti et al., 1989; Tsujii \& Seno, 1990; Nakamura et al., 1993). Thus, it was proposed (van der Heijden et al., 1995) that the murine splenic melanin is just an atavism of no systemic implications.

Van der Heijden et al. (1995) suggested that splenic melanin is a manifestation of cutaneous pigmentation of mice. Indeed, Weissman (1967) did not observe any splenic pigmentation in albino mice. Therefore, we decided to focus our study on melanin deposition in the spleen in relation to the hair cycle-dependent melanogenesis in C57BL/6 mice. The C57BL/6 mouse is an excellent model to study hair cycle, its physiology and pathology as well as systemic immune functions (Paus \& Cotsarelis, 1999; Stenn \& Paus, 2001; Mueller-Roever et al., 2001; Slominski et al., 2004; 2005). The process of hair shaft pigmentation is strictly coupled to the hair cycle (Slominski et al., 1991; Slominski \& Paus, 1993), a periodical turnover of cells which in anagen - the first phase of the cycle - create the pigmented hair shaft, and later undergo apoptosis in the next stage - catagen (Slominski et al., 2004; 2005). Also follicular melanocytes have been shown to undergo apoptosis at this stage (Tobin, 1998; Tobin et al., 1999). They may be replaced in the new hair cycle by a new pool of cells arisen from the appropriate progenitors (Nishimura et al., 2002), whereas the remaining apoptotic bodies which may contain melanin granules are phagocyted by Langerhans cells (Tobin, 1998; Slominski et al., 2004; 2005). The fate of this melanin is the most enigmatic process of hair pigmentation and melanocyte biology (Slominski et al., 1994; Tobin et al., 1999; Borovanský \& Elleder, 2003; Sulaimon \& Kitchell, 2003). Analogically to the case of lower vertebrates, one may hypothesize on the presence of melanophages which may transport some of the skin melanin to the place of its storage and further utilization. Melanin may be partially degraded in this process, because of its oxidative degradation in phagosomes of macrophages with the contribution of NADPH-oxidase, as suggested by Borovanský and Elleder (2003). Another possibility is deposition and degradation of melanin in organs destinated to detoxify and reutilize blood, such as spleen or liver. Finally, melanin may remain in the cells of origin, but even there its further degradation cannot be excluded (Stanka et al., 1988). In contrast, melanized hair coat is periodically or continuously shed, while in epidermis melanin is degraded to form "melanin dust" and is removed together with dead cells (Borovanský \& Elleder, 2003; Sulaimon \& Kitchell, 2003; Slominski et al., 2004; 2005).

It must also be noted that the potential of various leukocytes, including neutrophiles, macrophages and lymphocytes to serve as a system of melanin transport in vertebrates, being an alterna- tive or a supplement to the system of melanin transport within the "epidermal melanin units" was proposed already in the nineteenth century. In 1967 this idea was formulated by Wassermann, who also indirectly implied a similar notion concerning hair follicular pigmentation, and who connected both ideas with the phenomenon of visceral melanin deposition (Wasserman, 1967).

Hair cycling, including catagen and degradation of follicular melanocytes, is an immunologically-driven process (Paus \& Cotsarelis, 1999; Stenn \& Paus, 2001), which has systemic effects including, e.g., changes in the tempo of splenocyte proliferation (Slominski et al., 1997). Besides a direct engagement in the removal of follicular melanin phagocyted by the Langerhans cells in catagen, the spleen may participate indirectly in the cycle-related melanogenesis, or just passively reflect its activity. To address these questions we decided to focus on selected biophysical properties of skin and spleen melanin, in particular on their paramagnetic properties investigated by electron paramagnetic resonance (EPR) spectroscopy, being one of the most prominent, versatile and useful methods to investigate melanin in biological systems (Sarna \& Plonka, 2005; Slominski et al., 2005). Therefore, the following questions were addressed with the help of EPR spectroscopy: 1. Is the presence of the black pigment in spleen a rule, a tendency or perhaps a property only quantitatively correlated with the stage of animal development/ hair cycling stage? 2. Do the paramagnetic properties of the splenic pigment confirm its qualification as melanin, and what kind of melanin does it represent? 3. Is there a similarity between the splenic and hair/skin melanin? As the actual content and intensity of melanin production in skin changes in the telogen-anagen-catagen transition (Slominski \& Paus, 1993; Slominski et al., 2005), which may additionally interfere with the corresponding phenomena in the spleen, we have chosen to focus in this pilot study on telogen - the "resting" phase of hair cycle, when there is no melanogenesis in hair follicles (Slominski et al., 1991).

\section{MATERIAL AND METHODS}

Reagents and media. Phosphate-buffered saline (PBS) without calcium and magnesium was purchased at Wytwórnia Surowic i Szczepionek BIOMED (Lublin, Poland). Ketamine for narcosis $\left(\right.$ Ketanest $\left.^{\circledR}\right)$ - at ASTA Medica AG (Frankfurt, Germany), beeswax, gum rosin, and 3,4-dihydroxy $\mathrm{D}, \mathrm{L}-$ phenylalanine (D,L-dopa) - at Sigma-Aldrich (St. Louis, MO, USA).

Animals. Female inbred C57BL/6 mice 6-8week-old (Animal Breeding Facility, Silesian Medical Academy, Katowice-Ligota, Poland) were kept 
in social cages with free access to fresh water and standard rodent food. It is important to emphasize that the experimental groups consisted of closely related animals (sisters) of the same age, which entered the hair cycle almost on the same day (in the case of depilation-induced cycling - exactly on the same day and hour).

We focused our attention on the first and the second postnatal hair cycle (Paus et al., 1999; Mueller-Roever et al., 2001), and the experimental groups were sacrificed on different time points after entering telogen. The time schedule of the experiments and symbols of groups are shown in Fig. 1. Only mice with homogenous telogen hair follicle population were selected for experiments (Fig. 2A, B). The telogen stage of the hair cycle was recognized by homogenously pink color of the back skin, which can be appreciated particularly after depilation (Fig. 2 B) (Paus et al., 1990; Slominski et al., 1991; 1994). All the experiments were approved by the Permanent Rector Committee for Bioethics of Experiments on Animals (Jagiellonian University permission number 303/97), and performed according to the international rules accepted by the Polish Parliament.

Induction of hair growth. We investigated telogen skin after spontaneously induced hair growth, but we also analyzed some animals in which telogen followed artificially induced anagen (Figs. 1B, E and $2 \mathrm{~B}, \mathrm{C}$ ). The technique of anagen induction was based on the widely accepted depilation by application of melted 1:1 mixture of beeswax and gum rosin on the back skin of anesthetized animals (Ketamine, i.p. $0.75 \mathrm{mg} / \mathrm{mouse}$, in PBS), and removal together with the hair shafts after hardening (Mueller-Roever et al., 2001).

Hair, spleen and anagen skin preparation. Mice in ketamine narcosis with whole back skin in telogen (Fig. 2A), were shaved and their hair shafts were kept for the further EPR analysis. Hair shafts of yellow gerbils (a $40 \mathrm{mg}$ sample) served as a natural model material containing pheomelanin (Slominski et al., 2005; Plonka et al., 2003). The animals were killed by cervical dislocation, and spleens were removed. The presence, size and intensity of black spots (Fig. 2D-E) were estimated shortly before putting the organ into glass tubes, in which they were frozen, and after removal - measured by EPR. Finally, the mass of the spleens was recorded after the EPR measurement.

To obtain melanin-containing skin samples devoid of dead, highly pigmented and dry structures of hair shafts but consisting of both alive and melanized tissues, some mice in the late stage of depilation-induced anagen VI (day 15 after anagen in-

\section{GROUP}

A

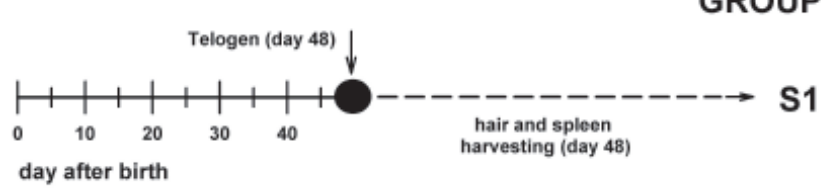

B
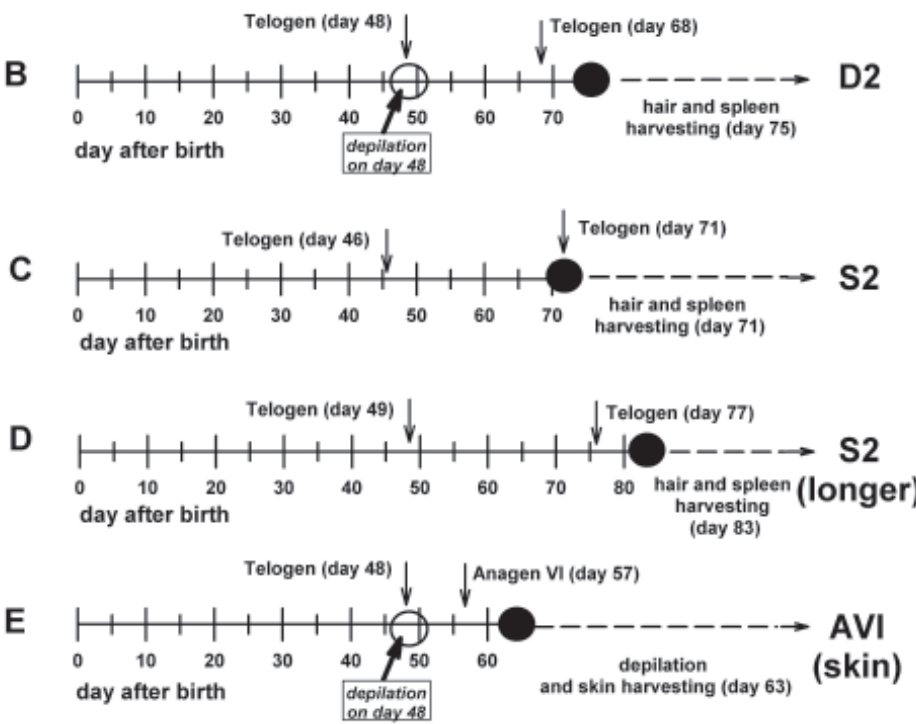

Figure 1. Experimental design.

A-D. Telogen groups where spleen and hair shafts were examined. E. Group from which depilated anagen VI skin containing melanin was obtained. Empty circles - the day of anagen induction by depilation. Filled circles - the day of autopsy and sample harvesting. Vertical arrows - days when the last mice from the group entered telogen, as judged by homogenously pink color of the back skin (A-E), and the day of the onset of anagen VI (E). 
duction, Fig. 1E) were anesthetized, depilated again (Fig. 2C), and sacrificed. The skin was tightly stuffed in standard glass tubes, frozen in liquid nitrogen, then pushed out from the tubes, and cut into two equal parts, so as to obtain samples of the same geometry (diameter $0.4 \pm 0.02 \mathrm{~cm}$; length $01.5 \pm 0.1 \mathrm{~cm}$ ) and mass $(0.2 \pm 0.003 \mathrm{~g})$.

Synthesis of dopa-melanin. Dopa-melanin was prepared by autooxidation of $\mathrm{D}, \mathrm{L}-\mathrm{dopa}$ (Felix et al., 1978a). An aqueous solution of D,L-dopa was bubbled with air for 4 days at ambient temperature, with the $\mathrm{pH}$ kept constant at $8\left(\mathrm{NH}_{4} \mathrm{OH}\right)$. Subsequently, the pigment formed was precipitated by lowering the $\mathrm{pH}$ to 3-3.5 $(\mathrm{HCl})$, purified with several washings with re-distilled water, and dialysed against re-distilled, deionized water for 4 days. It is worth noticing here that the term "melanin solution" should rather be replaced with "melanin suspension", because of difficulties in properly defining the type of solution formed by solubilized melanin (Crippa et al., 1989). The substance prepared by us contained about $10^{19}$ spins/mg dry mass, and it was used either in suspension $(13 \mathrm{mg} / \mathrm{ml}, \mathrm{pH}$ about 6.5$)$ or as air-dry powder samples $(8.2 \pm 0.1 \mathrm{mg})$.

EPR measurements and statistical analysis of the data. All the samples were analyzed in a quartz Dewar filled with liquid nitrogen, at $77 \mathrm{~K}$, using an EPR X-band $(9.18 \mathrm{GHz})$ spectrometer Varian E3 with a TE 102 rectangular resonant cavity. Icicles of skin and dopa-melanin suspension were pushed out of the tubes directly into the Dewar. Powder samples of dopa-melanin were measured in glass capillaries sealed by melting and solidifying their both ends, and spleens were measured in a similar way to the skin samples. The skin and melanin suspension samples, but not spleen and the powder melanin samples, had the same geometry. Samples of shaved hair shafts $(30 \pm 0.5 \mathrm{mg})$ had been stuffed directly in the Dewar flask before liquid nitrogen was poured in. A powder sample of 1,1-diphenyl2-picrylhydrazyl (DPPH) was used as a free-radical standard ( $g=2.0037 \pm 0.0002)$. All the measurements were performed at the magnetic field of $3260 \pm 50$ Gs. Other parameters of measurements were as follows: for qualitative comparison of the shape of the spectra (Fig. 3, Table 2) microwave power was 0.5 $\mathrm{mW}$ and modulation amplitude - 0.5 Gs. For qualitative comparisons (Table 1), modulation amplitude was raised to $5 \mathrm{Gs}$, and microwave power to $4 \mathrm{~mW}$, whereas gain was changed to 250000 . To register the power saturation curves (Fig. 4, Table 2) the microwave power was changed every single $\mathrm{dB}$ over the range of $31-8 \mathrm{~dB}(0.05-12 \mathrm{~mW})$, while the peak-topeak amplitude of the signal was measured, and the

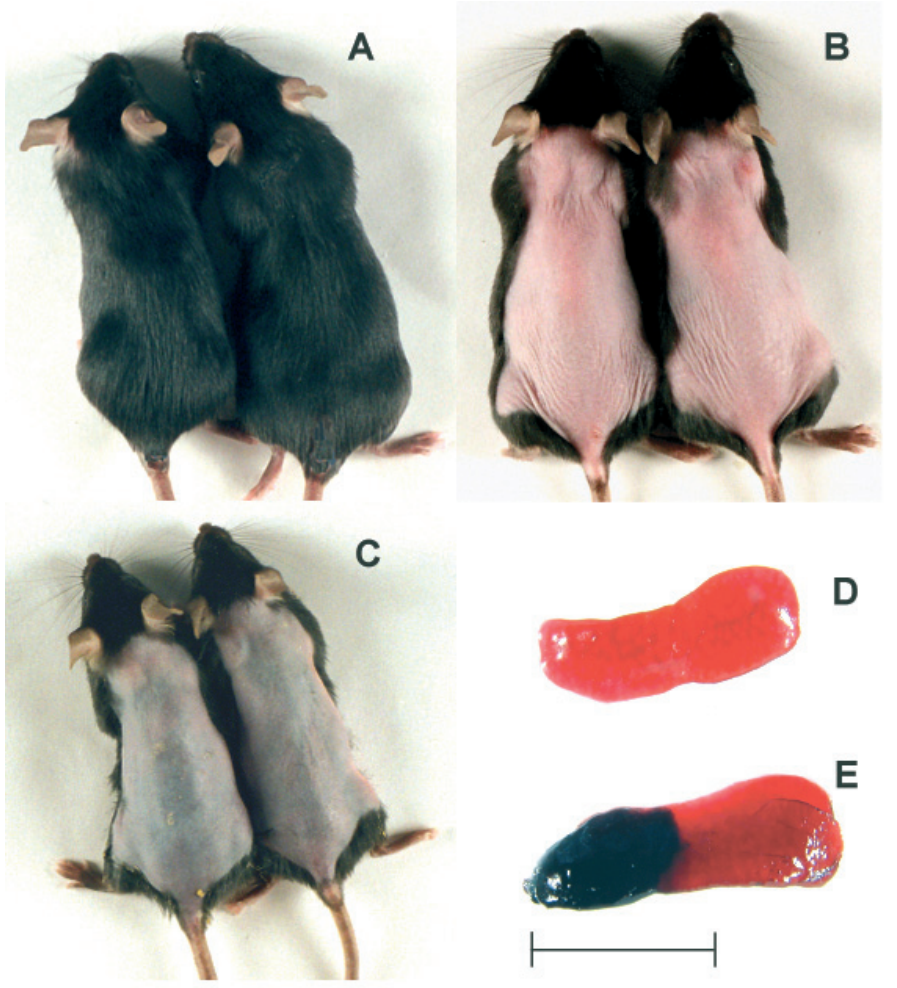

Figure 2. Macroscopic view of representative C57BL/6 animals and their spleens.

A. Non-depilated mice with whole back skin in telogen. B. Mice from group D2 shortly after depilation - note the homogenously pink back skin. C. Mice from group AVI (skin) on day 15 after the first depilation, and shortly after the second depilation. Note livid coloration of skin due to the on-going melanogenesis (compare with B). D. Typical red spleen without a black spot. E. Another spleen revealing a posterolateral melanotic spot. Scale bar $-1 \mathrm{~cm}$. 
Table 1. Appearance of black spots in the spleen in particular experimental groups of C57BL/6 mice in telogen.

Note the reverse correlation between the duration of telogen before spleen harvesting and the number of pigmented spleens in the group. The amount of melanin was estimated by the amplitude of the melanin EPR signal. Parameters of assay performed at $77 \mathrm{~K}$ : see Materials and Methods, receiver gain: 400000.

\begin{tabular}{lcccccc}
\hline $\begin{array}{l}\text { Number of the postnatal } \\
\text { hair cycle and the way } \\
\text { of its induction }\end{array}$ & $\begin{array}{l}\text { Group de- } \\
\text { signation }\end{array}$ & $\begin{array}{l}\text { Duration } \\
\text { of telogen } \\
\text { until harve- } \\
\text { sting (day) }\end{array}$ & $\begin{array}{l}\text { Total } \\
\text { number of } \\
\text { spleens }\end{array}$ & $\begin{array}{l}\text { Number } \\
\text { of sple- } \\
\text { ens with } \\
\text { a black } \\
\text { spot (\%) }\end{array}$ & $\begin{array}{l}\text { Number } \\
\text { of spleens } \\
\text { without the } \\
\text { black spots } \\
(\%)\end{array}$ & $\begin{array}{l}\text { Free radical EPR signal am- } \\
\text { plitude; ([a.u.], means from } \\
\text { pigmented spleens } \pm \text { S.D., or } \\
\text { value for the only pigmented } \\
\text { spleen in a group) }\end{array}$ \\
\hline 1 spontaneous & S1 & 0 & 6 & $3(50 \%)$ & $3(50 \%)$ & $3.08 \pm 0.08$ \\
2 spontaneous & S2 & 0 & 5 & $4(80 \%)$ & $1(20 \%)$ & $3.52 \pm 1.42$ \\
2 depilation-induced & D2 & 7 & 7 & $1(14.3 \%)$ & $6(85.7 \%)$ & 2.125 \\
2 spontaneous & S2(longer) & 6 & 7 & $1(14.3 \%)$ & $6(85.7 \%)$ & 3.3 \\
\hline
\end{tabular}

modulation amplitude was kept at 5 Gs. A lack of power saturation is usually manifested by a linear dependence of the signal amplitude on the square root of microwave power (Swartz \& Swartz, 1983). Each spectrum was collected in a digital form (1024 points per spectrum) for 120 or $200 \mathrm{~s}$ at a time constant of 0.1 or $0.3 \mathrm{~s}, 1-3$ times (for the weak signals of spleens and yellow gerbil hair $-5-7$ times) and averaged. To test the statistical significance of the observed differences, appropriate data were pooled, expressed as means \pm S.E.M. or means \pm S.D., and compared by two-tailed, independent Student's $t$ test for populations of equal- and non-equal variations, which was tested using the $F$ test.

\section{RESULTS AND DISCUSSION}

\section{Splenic melanin predominates in early telogen}

Murine C57BL/6 spleens (Fig. 2D) sometimes revealed black spots on their dorsal surface (Fig. $2 \mathrm{E})$. No variability or gradation of the color intensity (e.g. from bright through dark-grey to black) could be observed, only variability in the size of the spots was found, which were always homogenously black. The presence of a black spot was not the rule, and it never corresponded absolutely to the type of hair cycle induction - in the studied groups of telogen mice after the depilation-induced or spontaneous hair growth (Table 1) there were always some spleens with the dark spots besides spleens completely devoid of the pigment. Even in the group of the highest percentage of melanotic spleens, there was one animal with the spleen completely devoid of the pigment, and vice versa - in groups revealing amelanotic spleens there were single animals with melanotic spleen. Nevertheless, spleens from mice which had just entered telogen (Group S1, S2, see Fig. 1A, C) revealed more frequently the presence of melanin (Table 1) than those from mice which had already been in telogen for several days (Group D2, S2(longer) - Fig. 1B, D). This observation repre- sents the only correlation of the appearance of the dark spots with the hair cycling. This tendency was observed predominantly in animals with spontaneous hair cycling, which constituted 3 out of 4 experimental telogen groups (Fig. 1 A-D).

Although the most intensively pigmented spleen (of the highest amplitude of the free radical EPR signal, i.e. 5.675 a.u.) was found in the S2

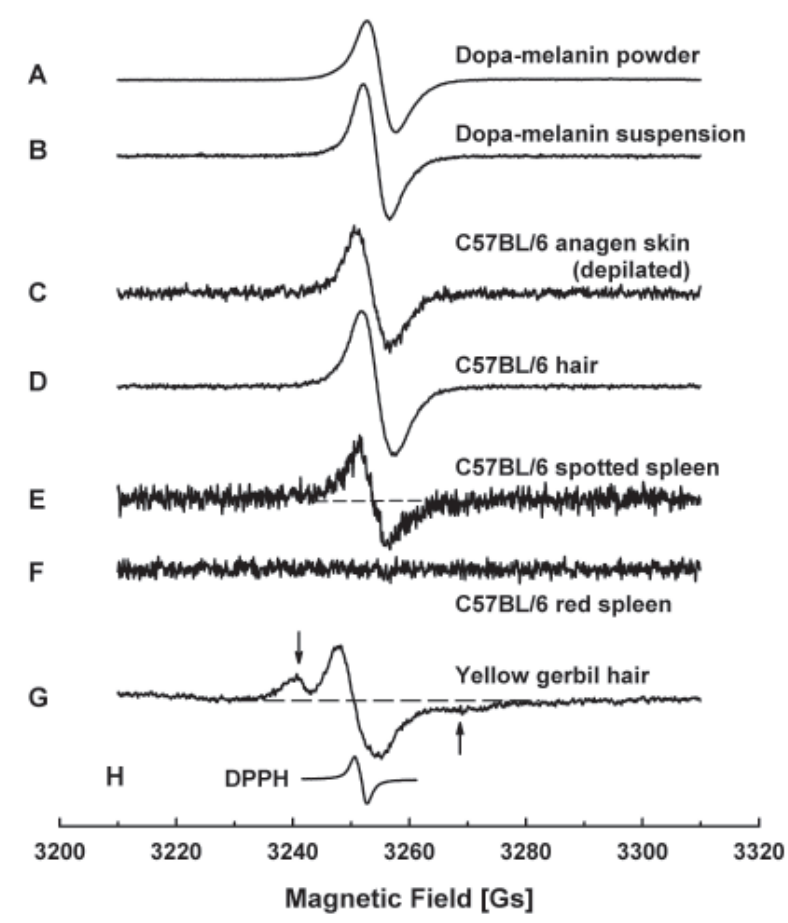

Figure 3. Comparison of EPR spectra of synthetic and natural pigmented materials used in the experiments.

Note the singlet line in the region of the DPPH and dopamelanin signal, present in the spectra of hair, skin and pigmented spleen of C57BL/6 mice, but not in the nonmelanized organ, which confirms the melanin-like character of the black splenic pigment. The lack of hyperfine splitting (arrows) well appreciable in the spectrum of yellow gerbil hair indicates the eumelanin type of pigment contained in the spotted spleen. All spectra were registered at $77 \mathrm{~K}$. Parameters of assay: see Material and Methods. Receiver gains: A - 40000, B -300000, C $1500000, \mathrm{D}-255000, \mathrm{E}-\mathrm{F}-7500000, \mathrm{G}-750000$. 
group, which had $80 \%$ spleens with the spots (Table $1)$, it was hardly possible to correlate the melanin contents with the percentage of melanized spleens in particular groups. For example (Table 1), 3.3 a.u. was found for another spleen of group S2, for one spleen of group S1, and for the only pigmented spleen of group S2(longer). The average mass of a pigmented spleen was slightly higher than the mass of non-melanized spleens, but this tendency was not significant $(P>0.2$; not shown).

The splenic pigment may represent melanin produced in the spleen due to the same activators which initiate melanogenesis in skin, due to accumulation of circulating intermediates of melanogenesis and/or melanin collected and degraded in other sites of melanin synthesis. In the black C57BL/6 a/a mice the main source for such melanin is the pool of follicular melanocytes, which periodically undergo apoptosis in catagen (Tobin, 1998; Tobin et al., 1999). Yet the appearance of dark spots in spleens ought to be considered as a stochastic phenomenon. It is a "yes or no" phenomenon which cannot be inferred from any behavioral or morphological evidence observed in the mice. Without laparotomy only the probability that a particular mouse has pigmented spleen can be determined on the base of the time which passed after the onset of telogen.

\section{Spleen, hair shafts and anagen skin of C57BL/6} mice contain eumelanin

Figure 3 shows EPR spectra of some pigmented materials obtained from rodents, including C57BL/6 mice, and the model melanins. In all the pigmented materials from mice, but not in non-pigmented spleens which revealed uniformly red coloration (Fig. 2D), a sharp singlet of a free radical character is seen in the region of both dopa-melanin and DPPH signals (Table 2, Fig. 3). Also the power saturability of the melanin signals is strong (Table 2, Fig. 4), which indicates their free-radical character (Felix et al., 1978b; Sarna \& Hyde, 1978).

The signal appeared as a slightly asymmetric singlet placed around $\mathrm{g}=2.003-2.004$, of the peakto-peak line-width of about 4.5-5.5 Gs, without any hyperfine splitting, which might indicate contribution of pheomelanin (Sealy et al., 1982; Slominski et al., 2005). Therefore, we can conclude that the type

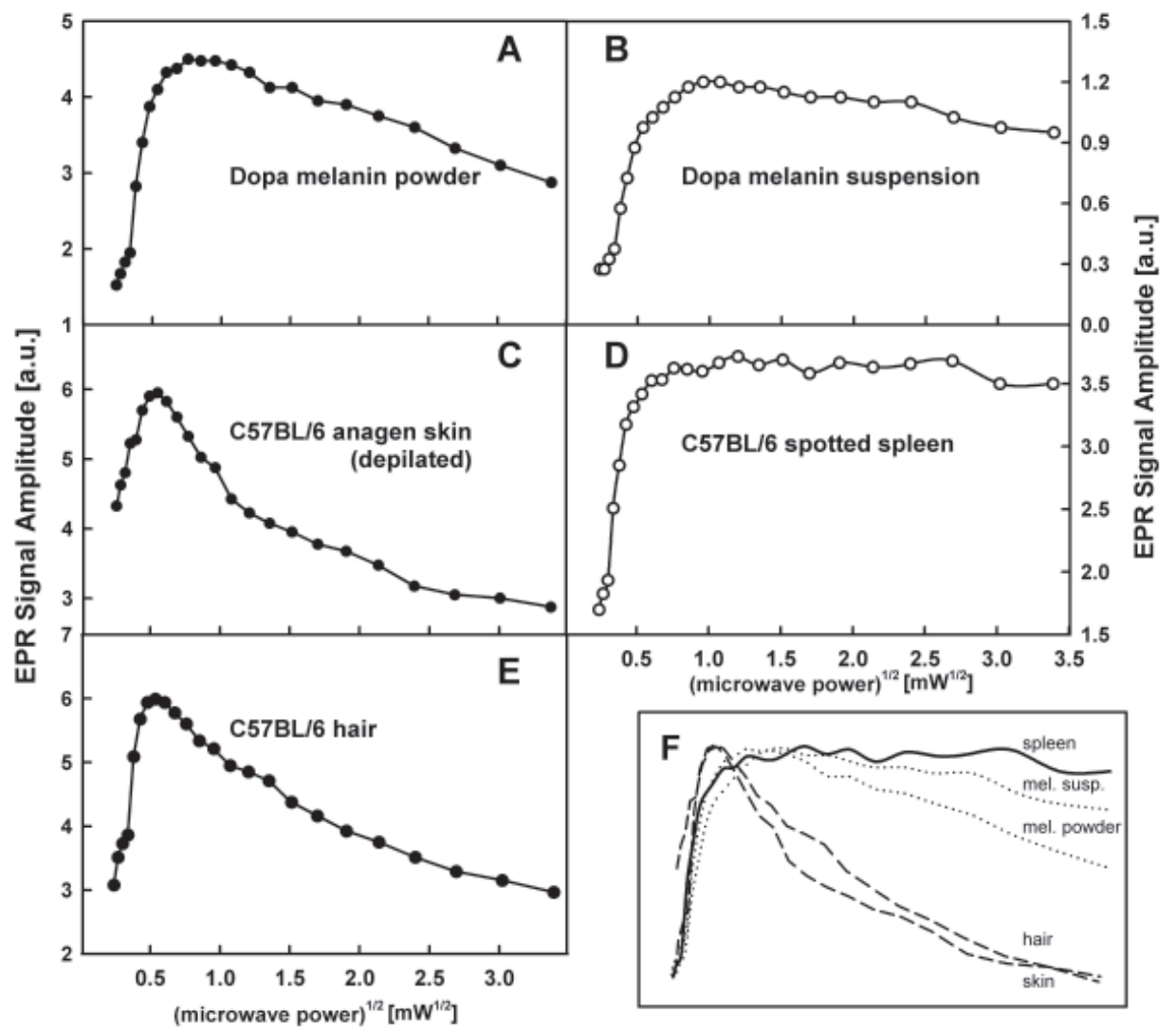

Figure 4. Saturation of the melanin EPR signal with microwave power.

A-B. Dopa melanin. C-D. Biological materials from C57BL/6 mice comprising normally hydrated tissues (C, depilated anagen VI skin; D, spleen with melanotic spot). E. Dry black hair shafts from C57BL/6 mice. All measurements were carried out at $77 \mathrm{~K}$. Parameters of assay: see Materials and Methods. Receiver gains: A-B - 2000, C - 125000, D $400000, \mathrm{E}-15000$. F. Superposition of curves A-E normalized for the same value of amplitude in maximum to compare the shapes of the saturation curves. 
Table 2. Parameters of the EPR signal of samples of pigments and biological materials obtained from C57BL/6 mice: peak-to-peak linewidth $(\Delta \mathrm{H})$ and the microwave power at which the saturation curve reaches its maximum (means of 3-6 samples \pm S.E.M.).

The difference between $\Delta \mathrm{H}$ for spleens and other murine materials was highly significant $(0.01>P \geq 0.001)$, and between the position of maxima - significant $(0.05>\mathrm{P} \geq 0.01)$. Parameters of assay performed at $77 \mathrm{~K}$ : see Fig. 3, 4, and Material and Methods.

\begin{tabular}{lcc}
\hline Material & $\Delta \mathrm{H}[\mathrm{Gs}]$ & $\begin{array}{l}\text { Maximum of the power } \\
\text { saturation curve } \\
{[\mathrm{mW}]([\mathrm{dB}])}\end{array}$ \\
\hline $\begin{array}{l}\text { Dopa melanin } \\
\text { (powder) }\end{array}$ & $4.74 \pm 0.11$ & $0.61 \pm 0.04$ \\
$(20.7 \pm 0.3)$ & $1.01 \pm 0.27$ \\
Dopa melanin & $4.45 \pm 0.12$ & $(18.5 \pm 1.0)$ \\
(suspension) & & $0.27 \pm 0.01$ \\
Depilated anagen & $5.52 \pm 0.05$ & $(24.3 \pm 0.1)$ \\
VI skin & $5.56 \pm 0.07$ & $0.29 \pm 0.01$ \\
Hair shafts & & $(24 \pm 0.1)$ \\
Spleens & $5.00 \pm 0.07$ & $0.84 \pm 0.46$ \\
with black spots & & $(19.3 \pm 2.0)$ \\
\hline
\end{tabular}

of melanin contained in the black-spotted spleens is eumelanin, the same as in the hair shafts of the animals. To our knowledge this is the first EPR confirmation of the presence of eumelanin in spleens of C57BL/6 mice.

Paramagnetic properties of splenic eumelanin indicate its heterogeneity and partial degradation

For the EPR study we had at our disposal two sets of pigmented skin-derived samples to compare their properties with the properties of pigmented spleens, namely the samples of dry hair shafts and the samples of pigmented skin. As the milieu of melanin in dry, dead matter of the hair shaft might differ from the conditions in melanosomes within live melanocytes, we chose skin of anagen VI phase of hair cycling (Fig. 1E) with hair shafts removed by additional depilation on day 15 after the first depilation (Fig. 2C). This skin contained only melanin closed in melanosomes of live melanocytes of hair follicles. The suspension and the powder sample of dopa melanin were synthetic equivalents of natural melanins (Slominski et al., 2005).

Comparison of the biological materials with synthetic dopa-melanin reveals that in both cases the properties of skin/hair melanin resemble more closely the powder samples of melanin than the water suspension. The latter reveals much narrower signals than the powder samples, and its saturation curve is shifted towards higher power, probably due to the presence of oxygen, as the solution was not deoxidized before freezing.

Splenic melanin also reveals narrower signals than the skin/hair pigment (Table 2). Their power saturability is also different - the saturation curves were in general much flatter in the case of spleens than for hair shafts or skin (Fig. 4C, D, E). The placement of the maximum was also shifted towards higher power, as compared to the hair/skin melanins. The poorer saturability and the flatter saturation curves of pigmented spleens, as compared to pigmented skin and hair shafts suggest higher heterogeneity of the local environments of the splenic paramagnetic centers (Swartz \& Swartz, 1983), and closer interactions with some rapidly relaxing paramagnetic centres, like dioxygen, iron (II), iron (III) or copper (II), which betrays heterogeneity within the pool of splenic melanin itself (Felix et al., 1978a). This finding supports the view that at least a fraction of splenic melanin undergoes degradation. It must be emphasized that, as the paramagnetism of splenic melanin is preserved, this degradation may primarily concern the proteins which are present along with melanin in melanosomes (Okazaki et al., 1985), and which are probably degraded first (Borovanský \& Elleder, 2003; Sulaimon \& Kitchell, 2003). Proteins may influence the linewidth as well as the power saturability (Sarna \& Hyde, 1978; Okazaki et al., 1985). This may to some degree explain the narrower signals of splenic melanin, which was also the case for the protein-free dopa-melanin, as compared to natural melanins. Proteolysis can be followed by oxidative degradation of melanin itself (Stanka et al., 1988; Borovanský \& Elleder, 2003).

Among the putative candidates for the function of "melanophages" responsible for transport and/or degradation of skin/hair follicle melanin, Langerhans cells seem the most important ones, due to their considerable migration potential and the role in scavenging the apoptotic bodies in catagen (Tobin, 1998; 1999). Other important cells potentially responsible for this phenomenon may be macrophages, due to their presence in hair follicles and their role in catagen remodeling (Parakkal, 1969a; 1969b). Their importance may increase with the aging of the animal (van der Heijen et al., 1995). Another type of cells suspected of transiently depositing ectopic melanin and particularly active during premature catagen induced by chemotherapy are noncortical keratinocytes (Tobin et al., 1999). Finally, macrophages and leukocytes (neutrophils and lymphocytes), have been suggested to participate in the process of "alternative melanin transport" in the vertebrate organism (Wassermann, 1967) to the place of its visceral deposition.

To our surprise, there were no evident qualitative differences between melanin from depilated anagen VI skin (Fig. 4C, Table 2) and that from hair shafts (Fig. 4E, Table 2), although we expected that the state of dehydratation of hair melanin, and the possible presence of oxygen, as compared with the melanosomes in live melanocytes and keratinocytes, 
might to some degree influence the spin-lattice relaxation properties of melanin paramagnetic centers, which might be reflected in the power saturability of the signals, and their linewidths (Sarna \& Hyde 1978, Felix et al., 1978b; Swartz \& Swartz 1983). This finding may be important for proper monitoring of the on-going melanogenesis in anagen of depilationinduced murine skin by EPR, in particular when the dry hair shaft is being produced (anagen IV-V) and stretches out of the skin (anagen VI) (Slominski et al., 1994; 1996; Plonka et al., 1995).

\section{CONCLUSIONS}

Using EPR spectroscopy we demonstrated here the presence of eumelanin in black-spotted spleens of C57BL/6 mice with similar biophysical properties as the pigment of black hair shafts and anagen skin melanocytes. However, the exact nature of the splenic eumelanin is different from that of skin/hair shaft melanin and reveals more heterogeneity, which can be to some degree explained by pigment degradation. The existence of melanin in the spleen is not the rule, although spleens of mice which had just entered telogen tended to contain pigmented spleens more often than was the case in animals which had been in telogen for a longer time.

\section{Acknowledgements}

This study was supported by the Center of Excellence in Molecular Biotechnology funded by the European Union and the Ministry of Science and Informatization, Poland (project "BIER", contract No. ICA1-CT-2000-70012, and SPUB-M 3018, Workpackage 6, to P.M.P.), and in part by grant PBZKBN-101/T09/2003/12 from the Ministry of Science and Informatization, Poland to P.M.P.

The authors are very grateful to Dr. Anna Wisniewska for her invaluable help with preparation of the manuscript.

\section{REFERENCES}

Borovanský J, Elleder M (2003) Melanosome degradation: fact or fiction. Pigment Cell Res 16: 280-286.

Cicero R, Sciuto S, Chillemi R, Sichel G (1982) Melanosynthesis in the Kupffer cells of Amphibia. Comp Biochem Physiol 73A: 477-479.

Cicero R, Mallardi A, Maida I, Gallone A, Pintucci G (1989) Melanogenesis in the pigment cells of Rana esculenta L. liver: evidence for tyrosinase-like activity in the melanosome protein fraction. Pigment Cell Res 2: 100-108.

Crippa R, Horak V, Prota G, Svoronos P, Wolfram L (1989) Chemistry of melanins. In The Alkaloids. Brossi A, ed, vol 36, pp 253-323. Academic Press, New York.
Felix CC, Hyde JS, Sarna T, Sealy RC (1978a) Interactions of melanin with metal ions. Electron spin resonance evidence for chelate complexes of metal ions with free radicals. J Am Chem Soc 100: 3922-3926.

Felix CC, Hyde JS, Sarna T, Sealy RC (1978b) Melanin photoreactions in aerated media. Electron spin resonance evidences for production of superoxide and hydrogen peroxide. Biochem Biophys Res Commun 84: 335-341.

Mueller-Roever S, Handjiski B, van der Veen C, Eichmuller S, Foitzik K, McKay IA, Stenn KS, Paus R (2001) A comprehensive guide for the accurate classification of murine hair follicles in distinct hair cycle stages. I Invest Dermatol 117: 3-15.

Nakamura H, Shimozawa A, Kikuchi S-I (1993) Melanomacrophage centra-like structure in the heart of the Medaka, Oryzias latipes. Ann Anat 175: 59-63.

Nishimura EK, Jordan SA, Oshima H, Yoshida H, Osawa M, Moriyama M, Jackson IJ, Barrandon Y, Miyachi Y, Nishikawa S (2002) Dominant role of the niche in melanocyte stem-cell fate determination. Nature 416: 854-860.

Okazaki M, Kuwata K, Miki Y, Shiga S, Shiga T (1985) Electron spin relaxation of synthetic melanin and melanin-containing human tissues as studied by electron spin echo and electron spin resonance. Arch Biochem Biophys 242: 197-205.

Parakkal PF (1969a) Involvement of macrophages in collagen resorption. J Cell Biol 41: 345-354.

Parakkal PF (1969b) Role of macrophages in collagen resorption during hair growth cycle. J Ultrastruct Res 29: 210-217.

Paus R, Cotsarelis G (1999) The biology of hair follicles. N Engl J Med 341: 491-497.

Plonka P, Plonka B, Paus R (1995) Biophysical monitoring of melanogenesis as a tool for pigment and hair research. Arch Dermatol Res 287: 687-690.

Plonka PM, Slominski AT, Pajak S, Urbanska K (2003) Transplantable melanomas in gerbils (Meriones unguiculatus). II - Melanogenesis. Exp Dermatol 12, 356-364.

Sarna T, Hyde JS (1978) Electron spin-lattice relaxation times of melanin. J Chem Phys 69: 1945-1948.

Sarna T, Plonka PM (2005) Biophysical studies of melanin: paramagnetic, ion-exchange and redox properties of melanin pigments and their photoreactivity. In Biomedical ESR. Biological Magnetic Resonance Series. Eaton SS, Eaton GR, Berliner LJ, eds, vol 23, pp 125-146. Kluwer Academic Publishers, The Netherlands, New York, Boston.

Sealy R, Hyde J, Felix C, Menon I, Prota G (1982) Eumelanins and phaeomelanins: characterization by electron spin resonance spectroscopy. Science 217: 545-547.

Sichel G (1988) Biosynthesis and function of melanins in hepatic pigmentary system. Pigment Cell Res 1: 250258.

Slominski A, Goodman-Snitkoff G, Maurer M, Paus R (1997) Hair cycle-associated changes in splenocyte proliferation. In Vivo 11: 101-102.

Slominski A, Paus R (1993) Melanogenesis is coupled to murine anagen: toward new concepts for the role of melanocytes and the regulation of melanogenesis in hair growth. J Invest Dermatol 101: 90S-97S.

Slominski A, Paus R, Costantino R (1991) Differential expression and activity of melanogenesis-related proteins during induced hair growth in mice. J Invest Dermatol 96: 172-179.

Slominski A, Paus R, Plonka P, Chakraborty A, Maurer M, Pruski D, Lukiewicz S (1994) Melanogenesis during the anagen-catagen-telogen transformation of the murine hair cycle. J Invest Dermatol 102: 862-869. 
Slominski A, Paus R, Plonka P, Handjiski B, Maurer M, Chakraborty A, Mihm MCM Jr (1996) Pharmacological disruption of hair follicle pigmentation by cyclophosphamide as a model for studying the melanocyte response to and recovery from cytotoxic drug damage in situ. J Invest Dermatol 106: 1203-1211.

Slominski A, Tobin DJ, Shibahara S, Wortsman J (2004) Melanin pigmentation in mammalian skin and its hormonal regulation. Physiol Rev 84: 1155-1228.

Slominski A, Wortsman J, Plonka PM, Schallreuter KU, Paus R, Tobin DJ (2005) Hair follicle pigmentation. J Invest Dermatol 124: 13-21.

Stanka P, Bargsten G, Sahlmann B (1988) On the formation and degradation of melanosomes in smooth muscle cells: electron microscopic investigation on the $\mathrm{m}$ sphincter pupillae of the rat. Pigment Cell Res 1: 358 360.

Stenn KS, Paus R (2001) Controls of hair follicle cycling. Physiol Rev 81: 449-494.

Sulaimon SS, Kitchell BE (2003) The biology of melanocytes. Vet Dermatol 14: 57-65.

Swartz HM, Swartz SM (1983) Biochemical and biophysical applications of electron spin resonance. In Methods of Biochemical Analysis. Glick D, ed, vol 29, pp 207-323. John Wiley \& Sons, Inc., New York.
Tobin DJ (1998) A possible role for Langerhans cells in the removal of melanin from early catagen hair follicle. $\mathrm{Br}$ J Dermatol 168: 795-798.

Tobin DJ, Slominski A, Botchkarev V, Paus R (1999) The fate of hair follicle melanocytes during the hair growth cycle. J Invest Dermatol Symp Proc 4: 323-332.

Tsujii T, Seno S (1990) Melano-macrophage centers in the aglomerular kidney of the sea horse (Teleosts): morphologic studies on its formation and possible functions. Anat Rec 226: 460-470.

Van der Heijen A, van Dijk JE, Lemmens AG, Beynen AC (1995) Spleen pigmentation in young C57BL mice is caused by accumulation of melanin. Labor Anim 29: 459-463.

Wassermann HP (1967) Extension of the concept "vertebrate epidermal melanin unit" to embrace visceral pigmentation and leucocytic melanin transport. Nature 213: $282-283$.

Weissman I (1967) Genetic and histochemical studies on mouse spleen black spots. Nature 215: 315.

Zuasti A, Jara JR, Ferrer C, Solano F (1989) Occurence of melanin granules and melanosynthesis in the kidney of Sparus auratus. Pigment Cell Res 2: 93-99. 